\title{
The rheology of a suspension of nearly spherical particles subject to Brownian rotations
}

\author{
By L. G. LEAL \\ Chemical Engineering, California Institute of Technology \\ AND E. J. HINCH \\ Department of Applied Mathematics and Theoretical Physics, \\ University of Cambridge
}

(Received 1 March 1972)

A set of constitutive equations, valid for arbitrary linear bulk flows, is derived for a dilute suspension of nearly spherical, rigid particles which are subject to rotary Brownian couples. These constitutive equations are subsequently applied to find the resulting stress patterns for a variety of time-dependent bulk flow fields. The rheological responses are found to exhibit many of the same qualitative features as have been observed in recent experimental investigations of polymeric solutions and other complex materials.

\section{Introduction}

The bulk stress of a suspension of rigid particles in a Newtonian fluid is sensitive to the orientation distribution of the particles. In this paper we consider a dilute suspension of spheroids under circumstances in which the orientation distribution is determined by the competition of random disorientating Brownian couples acting against the tendency towards a preferred alignment which results from the action of the bulk flow.

The earliest theoretical investigations of this model were restricted to steady bulk shear flow and small deformation rates, and used energy dissipation arguments which yield only the effective viscosity (cf. Boeder 1932; Burgers 1938). In 1962, Giesekus calculated the full bulk stress tensor for steady shear in the limit of low shear rates. The low deformation rate results were partially extended to higher shear rates by Scheraga (1955) and Takserman-Krozer \& Ziabicki (1963) by the numerical evaluation of a larger number of terms in an asymptotic expansion about the low deformation rate limit, and by Stewart \& Sørensen (1972) by the application of Galerkin's methods. The extension to time-dependent and other types of flow has not yet received much attention in spite of its obvious importance, the main previous works being the investigations of oscillating shear flow by Kirkwood \& Auer (1951) and Kirkwood \& Plock (1956), Giesekus's (1962) restatement of his steady shear results in a second-order form applicable to all flow fields, and Takserman-Krozer \& Ziabicki's (1963) study of steady axisymmetric extensional flow. 
Recently the present authors have discussed the rheological (Hinch \& Leal 1972) and the rheo-optical (Leal \& Hinch 1972) properties of a dilute suspension of spheroids for steady shear flow $\dagger$ in an exhaustive set of limiting cases which encompasses the whole range of flow strengths. For particle aspect ratios (the ratio of the major to minor semi-diameters) differing significantly from unity, approximate solutions were obtained for weak shear flow (a restatement of Giesekus 1962), for strong shear flow (a problem commenced in Leal \& Hinch 1971 ) and finally for a new intermediate range of flow strengths when the particle aspect ratio is extreme. As the shear rate is increased from zero, the microstructure, which is statistically isotropic in the rest state, becomes increasingly anisotropic: the orientation of the particles becomes dominated by the bulk flow rather than Brownian motions. This shear-dependent orientational anisotropy results in non-Newtonian rheological behaviour. For example, the effective viscosity of the suspension is found to exhibit shear thinning, decreasing from a constant value in the limit of small shear rate to a second constant value as the orientation distribution achieves its large shear rate limiting form. The principal and secondary normal stress differences are non-zero, of opposite sign, unequal in magnitude and for low rates of shear, increase quadratically in the shear rate, finally tending to constant values in the limit of high shear rates.

Because the calculations were restricted to various limiting cases of possible shear rate, as noted above, no generally applicable constitutive equation could be obtained except in the special case of near-spheres. In this limit, an explicit nonlinear representation of the bulk stress which is valid over the complete range of shear rates was found. Particularly significant is the fact that the near-sphere suspension was found to differ from the general case only in the relative magnitude of the predicted non-Newtonian effects, not in the qualitative manner of its behaviour. Since an explicit representation which is valid for arbitrary shear rates is possible, the near-sphere model would thus seem to offer a potentially valuable system for examining at least the qualitative aspects of the suspension behaviour in more general bulk flows.

In this paper we derive a simple set of rheological constitutive equations for a general-tensorial time-dependent deformation gradient. The asymptotic expansion used for the particle orientation is a generalization of that given by Peterlin (1938). Specifically, the limit considered is that of the aspect ratio tending to unity, for a fixed flow strength which can be of arbitrary magnitude compared with the Brownian effects. As we shall see, however, this form of the double limit can be relaxed for certain flow forms, e.g. for shear but not for pure strains. In the latter case the approximation does exclude some qualitative features of the suspension rheology: for example, there is no constant limit effective viscosity for large strain rates within our model; instead, with increasing strain rate, the viscosity increases until the validity constraints are violated.

To help comprehend the constitutive equation, which contains both nonlinearity and a memory effect, we subsequently apply it to several illustrative deformation histories to deduce the response of the suspension. The particular

$\dagger$ A generalization of these shear flow results to a wider class of steady two-dimensional flows was also noted. 
flow examples we study are: simple shear - the steady case, the start-up to and the relaxation from the steady case, small amplitude oscillations, and small amplitude oscillations superimposed on a steady arbitrary strength shear; axially symmetric strain - the steady case, the Heaviside start-up and the ramp start-up; a steady shear with superimposed small amplitude oscillations of a transverse shear; and finally the steady Maxwell orthogonal rheometer flow. We shall remark on the similarity of many of the qualitative features of this suspension with experimentally observed behaviour of quite different complex materials. We hope that our simple model might be useful in beginning to understand the physics of the orientation of the microstructure, which we believe is common to many complex materials.

The only other general study of a dilute suspension of axially symmetric particles subject to Brownian couples is that of Bird, Warner \& Evans (1971), who investigated dumb-bells in the limit of weak flow strengths. Their double limit is that of infinite aspect ratios, followed by dominant Brownian effects.

\section{The constitutive relation}

We consider a dilute suspension of identical rigid spheroids which are free from externally applied couples and forces but are subjected to rotary Brownian movements. We understand dilute to imply, as usual, that the suspended particles are hydrodynamically independent. We therefore begin by looking at the free motion of a single spheroid subjected to the external flow

$$
\mathbf{u}(\mathbf{x}, t)=\boldsymbol{\Gamma}(t) . \mathbf{x}
$$

The velocity gradient tensor may be expressed as

$$
\boldsymbol{\Gamma}=\mathbf{E}+\boldsymbol{\Omega}, \quad \mathbf{E}^{T}=\mathbf{E}, \quad \boldsymbol{\Omega}^{T}=-\boldsymbol{\Omega},
$$

where $\mathbf{E}(t)$ is the rate-of-strain tensor and $\boldsymbol{\Omega}(t)$ the vorticity tensor. The more common vorticity vector $\boldsymbol{\Omega}$ is given by

$$
\Omega_{i}=-\frac{1}{2} \epsilon_{i j k} \Omega_{j k} .
$$

We denote the particle aspect ratio by $r(r>1$ for a prolate spheroid) and specify the orientation of the spheroid by the direction of a unit vector $p$ coaxial with its axis of revolution.

In the absence of inertial, Brownian motion and particle interaction effects, Jeffery's (1922) solution shows the angular velocity $\boldsymbol{\Omega}^{\prime}$ of the spheroid to be given by

$$
\boldsymbol{\Omega}^{\prime}=\boldsymbol{\Omega}+\frac{r^{2}-1}{r^{2}+1} \mathbf{p} \wedge(\mathbf{E} \cdot \mathbf{p})
$$

Hence, the time change in orientation is

$$
\dot{\mathbf{p}}=\mathbf{\Omega} \cdot \mathbf{p}+\frac{r^{2}-1}{r^{2}+1}[\mathbf{E} \cdot \mathbf{p}-\mathbf{p}(\mathbf{p} \cdot \mathbf{E} \cdot \mathbf{p})]
$$

Bretherton (1962) has noted that nearly all axially symmetric particles rotate according to this expression provided that $r$ is replaced by an appropriate aspect ratio. 
As is customary, the statistics of a particle's orientation are described using the probability density function $N(\mathbf{p})$, defined such that the probability of the particle being oriented within an elementary solid angle $d \varpi$ of $\varpi$ is $N d \varpi$. The space of all possible orientations is identical to the surface of a unit sphere. Since the particle must have some orientation at each instant, the probability density is normalized by

$$
\int_{\varpi} N d w=1
$$

Following Burgers (1938) and subsequent workers, temporal changes in $N$ are governed by the equation

$$
\partial N / \partial t+\nabla \cdot \mathbf{F}=\mathbf{0}
$$

expressing the conservation of probability density with a flux $\mathbf{F}$ of particle probability in the orientation space given by

$$
\mathbf{F}=\dot{\mathbf{p}} N-D \nabla N
$$

where $D$ is the rotational Brownian coefficient, which has been defined for spheroids in Leal \& Hinch (1971). Combining the above equations gives

$$
\partial N / \partial t+\nabla \cdot(\dot{\mathbf{p}} N)=D \nabla^{2} N
$$

or in terms of derivatives with respect to $\mathbf{p}$

$$
\begin{aligned}
\frac{\partial N}{\partial t}-\mathbf{p} \cdot \boldsymbol{\Omega} \cdot \frac{\partial N}{\partial \mathbf{p}}-\frac{r^{2}-\mathbf{1}}{r^{2}+1}\left[\mathbf{p} . \mathbf{E} \cdot \mathbf{p}\left(3 N+\mathbf{p} \cdot \frac{\partial N}{\partial \mathbf{p}}\right)-\mathbf{p} \cdot \mathbf{E} \cdot \frac{\partial N}{\partial \mathbf{p}}\right] \\
=D\left[(\mathbf{I}-\mathbf{p p}): \frac{\partial^{2} N}{\partial \mathbf{p} \partial \mathbf{p}}-2 \mathbf{p} \cdot \frac{\partial N}{\partial \mathbf{p}}\right] .
\end{aligned}
$$

We follow Giesekus (1962) and split the bulk (volume averaged) stress into two parts:

$$
\boldsymbol{\sigma}=(-p \mathbf{I}+2 \mu \mathbf{E})+\boldsymbol{\sigma}^{\prime}
$$

the first representing the stress which would occur in the absence of particles and the second, $\sigma^{\prime}$, representing the particle contribution. The particle contribution $\boldsymbol{\sigma}^{\prime}$ to the bulk stress is itself conveniently written as the sum of two parts: $\boldsymbol{\sigma}^{s}$, the strain-stress representing the contribution from a particle which is undisturbed by Brownian motion except insofar as it governs the orientation distribution, and $\sigma^{D}$, the diffusion-stress representing the contribution arising from the effective particle rotation associated with the rotary Brownian diffusion process.

The strain-stress $\sigma^{s}$ for a particle at one particular orientation is evaluated following Batchelor (1970). The contributions from each possible orientation are found, weighted according to the probability of a particle being so aligned and finally summed. Further details of the calculation may be found in Hinch \& Leal (1972). The result for near-spheres is

$$
\boldsymbol{\sigma}^{s}=2 \mu \Phi\left\{\frac{395}{147} \epsilon^{2}\langle\mathbf{p p p p}\rangle: \mathbf{E}+\left(\frac{15}{14} \epsilon-\frac{895}{588} \epsilon^{2}\right)[\langle\mathbf{p p}\rangle . \mathbf{E}+\mathbf{E} .\langle\mathbf{p p}\rangle]+\frac{5}{2}\left(1-\frac{2}{7} \epsilon+\frac{1}{3} \epsilon^{2}\right) \mathbf{E}\right\}
$$


where $\epsilon=r-1 \ll 1$, $\Phi$ is the volume concentration of suspended particles and $\mu$ the viscosity of the ambient suspending liquid. The pointed brackets denote an average over the orientation space of the included function, i.e.,

$$
\langle M\rangle=\int_{\pi} M(\mathbf{p}) N(\mathbf{p}) d \sigma .
$$

In a similar manner the diffusion-stress may be evaluated, with the result

$$
\boldsymbol{\sigma}^{D}=2 \mu \Phi\{9 D \epsilon\langle\mathbf{p} \mathbf{p}\rangle\} .
$$

These expressions (2) and (3) are equivalent to those of Giesekus (1962) in the case of a shear flow, as well as the equations (9) and (10) of Hinch \& Leal (1972). The stress contribution of any particle of revolution will be qualitatively similar but with the shape-dependent coefficients differently related to the effective particle aspect ratio.

In order to evaluate the averages $\langle>$ in the expressions for the bulk stress, it is necessary to derive a solution of (1) for the orientation distribution function. An approximate series solution valid for nearly spherical spheroids in a steady simple shear flow was given by Peterlin (1938). Peterlin \& Stuart (1939) used this solution to determine the effective viscosity of the suspension in the limit of strong Brownian motion. Later this unnecessary restriction to strong Brownian motion was removed in calculations of certain optical properties (Sadron 1953) and of the bulk stress for steady shear flows (Hinch \& Leal 1972). In addition, Scheraga (1955) included a very large number of terms of Peterlin's series solution to calculate the effective viscosity for $\frac{1}{300} \leqslant r \leqslant 300$ and $0 \leqslant \gamma / D \leqslant 60$. In the following, Peterlin's expansion is applied to the general linear timedependent flow.

We begin with the formal expansion in the small parameter $\left(r^{2}-1\right) /\left(r^{2}+1\right)$ :

$$
N=N_{0}+\frac{r^{2}-1}{r^{2}+1} N_{1}+\cdots
$$

The strict double limit we are taking here is $r \rightarrow 1$ with $(1 / D) \mathbf{E},(1 / D) \boldsymbol{\Omega}$ fixed. However, it can be shown that this may be relaxed to requiring the eigenvalues of the matrix

$$
\frac{1}{D}\left(\frac{r^{2}-1}{r^{2}+1} \mathbf{E}+\boldsymbol{\Omega}\right)
$$

to have small positive parts. In the case of shear flow this reduces to the unconstrained limit $r \rightarrow 1$, but for pure strains it is necessary to keep $|\mathbf{E}|\left(r^{2}-1\right) / D\left(r^{2}+1\right)$ small.

Substitution of the expansion into the (1) generates the problems

$$
\begin{aligned}
\frac{\partial N_{n}}{\partial t}-\mathbf{p} \cdot \boldsymbol{\Omega} \cdot \frac{\partial N_{n}}{\partial \mathbf{p}}-D\left[(\mathbf{I}-\mathbf{p p}): \frac{\partial^{2} N_{n}}{\partial \mathbf{p} \partial \mathbf{p}}-2 \mathbf{p} \cdot \frac{\partial N_{n}}{\partial \mathbf{p}}\right] \\
\quad=\left\{\begin{array}{cc}
\mathbf{0} & (n=0), \\
\mathbf{p} \cdot \mathbf{E} \cdot \mathbf{p}\left(3 N_{n-1}+\mathbf{p} \cdot \frac{\partial N_{n-1}}{\partial \mathbf{p}}\right)-\mathbf{p} \cdot \mathbf{E} \cdot \frac{\partial N_{n-1}}{\partial \mathbf{p}} & (n>0) \cdot\}
\end{array}\right.
\end{aligned}
$$


At the zeroth order the particles are spheres. Any central axis of a sphere can be an axis of revolution, so that the orientation of 'the' principal axis must be purely random, i.e. $N_{0}=1 / 4 \pi$. The forcing of $N_{1}$ by the right-hand side of (4) is thus calculated as

$$
(3 / 4 \pi) \text { p.E.p. }
$$

This suggests trying a general quadratic form for $N_{1}$ :

$$
N_{1}=(1 / 4 \pi) \mathbf{p} \cdot \mathbf{A} \cdot \mathbf{p},
$$

where $\mathbf{A}(t)$ is symmetric and traceless. The latter condition is a consequence of the normalization. Substituting this form for $N_{1}$ into (4) yields

$$
\mathscr{D A} / \mathscr{D} t+6 D \mathbf{A}=3 \mathbf{E},
$$

where $\mathscr{D} / \mathscr{D} t$ is the Jaumann derivative:

$$
\mathscr{D A} / \mathscr{D} t=\partial A / \partial t+\mathbf{A} \cdot \mathbf{\Omega}-\boldsymbol{\Omega} . \mathbf{A} .
$$

The angle averages for the bulk stress can now be found to sufficient accuracy in terms of A. Two identities for the projection averages are needed:

$$
\begin{gathered}
\int_{\varpi} p_{i} p_{j} d \varpi=\frac{4 \pi}{3} \delta_{i j}, \\
\text { Thus, e.g. } \quad\langle\mathbf{p} \mathbf{p}\rangle=\frac{1}{3} \mathbf{I}+\frac{2}{15} \frac{r^{2}-1}{r^{2}+1} \mathbf{A}+O\left(\frac{r^{2}-1}{r^{2}+1}\right)^{2} .
\end{gathered}
$$

The bulk stress contribution due to the presence of the suspended particles is then found from (2) and (3) to be

$$
\boldsymbol{\sigma}^{\prime}=2 \mu \Omega\left\{\frac{5}{2} \mathbf{E}+\epsilon^{2}\left[\frac{26}{147} \mathbf{E}+\frac{6}{5} D \mathbf{A}+\frac{1}{7}\left(\mathbf{E} \cdot \mathbf{A}+\mathbf{A} \cdot \mathbf{E}-\frac{2}{3} \mathbf{I}(\mathbf{A}: \mathbf{E})\right)\right]+O\left(\epsilon^{3}\right)\right\} .
$$

The equations (5) and (6) represent the constitutive relation for the suspension and are the main results of this section. From a continuum-mechanics point of view, our model is an example of a simple class of anisotropic fluids. However, in distinction from the approach of continuum mechanics, each term of the model's constitutive equations can be related to a specific microstructural effect, and further, our analysis has determined explicitly the material coefficients. We believe that the natural appearance of the Jaumann derivative in this expression and other expressions in suspension rheology (cf. Frankel \& Acrivos 1970) is significant in view of the apparent inability of general continuum mechanies to distinguish between this and other invariant time derivatives. It will be noted that the stress deviates from the Einstein value of $\Phi \frac{5}{2} 2 \mu \mathbf{E}$ by quadratically small terms. In our opinion, the value of the results (5) and (6) is not primarily their potential technical application but the explicit representation of a complex material. The memory of the suspension enters with the time derivative of (5). The nonlinear behaviour is present in the second $6 D \mathbf{A}$ term of (5) compared with the Jaumann derivative $(\mathscr{D} \mathbf{A} / \mathscr{D} t)$ (doubling $\mathbf{\Gamma}$ will not double $\mathbf{A}$ ), and in the product of $\mathbf{E}$ and $\mathbf{A}$ in (6). The nonlinear effect will be shown in a following section 
to yield a strain-thickening viscosity. As was indicated previously, the combined memory and nonlinear effects in shear flow produce a shear-thinning viscosity and normal stress differences (see the following section and also Hinch \& Leal 1972), as well as a lag in the material response to imposed temporal variations in the bulk flow.

Quite similar constitutiverelations to (5) and (6) have been found in suspensions of other near-spherical particles. Goddard \& Miller (1967) have an elasticity resisting the distortion of the particle shape instead of Brownian diffusion resisting anisotropy in the orientation distribution, whereas Frankel \& Acrivos (1970) have a surface tension playing a similar role for a suspension of drops. These other studies were mostly restricted to low bulk deformation rates.

In the following sections, we apply the relations (5) and (6) to several specific bulk flows in order to indicate the types of material response which this nearsphere suspension is capable of producing. We shall restrict our attention to simple flows which could be used in principle to actually measure the response of the materials. An alternative scheme to that followed here would be to exploit the calculus of the Jaumann derivative, as developed by Goddard \& Miller (1966), invert (5) and hence achieve an explicit representation for the stress in terms of the history of the strain rate. This expression for general flows is complex, involving material integrals of the product of the material matrizant tensor with the strain rate tensor, and is still not easily interpretable without reverting to specific flows.

There are two approximate solutions of (5) and (6) for general flows which are relatively simple. When the flow is weak and slowly varying the constitutive equations reduce to the second-order fluid form

$$
\boldsymbol{\sigma}^{\prime}=2 \mu \Phi\left\{\frac{5}{2} \mathbf{E}+\epsilon^{2}\left[\frac{571}{735} \mathbf{E}-\frac{1}{10} \frac{1}{D} \frac{\mathscr{D} \mathbf{E}}{\mathscr{D} t}+\frac{1}{7} \frac{1}{D}\left(\mathbf{E} . \mathbf{E}-\frac{1}{3} \mathbf{I}[\mathbf{E}: \mathbf{E}]\right)\right]\right\}
$$

the next term in the approximation scheme is the third-order fluid correction

$$
+2 \mu \Phi \epsilon^{2}\left\{\frac{1}{60} \frac{1}{D^{2}} \frac{\mathscr{D}^{2}}{\mathscr{D} t^{2}} \mathbf{E}-\frac{1}{84} \frac{1}{D^{2}} \frac{\mathscr{D}}{\mathscr{D} t}\left(\mathbf{E} . \mathbf{E}-\frac{1}{3} \mathbf{I}(\mathbf{E}: \mathbf{E})\right)\right\}
$$

For weaker flow strengths but with no limitation on the time changes the appropriate approximation is known as the linear viscoelastic form:

$$
\left(\frac{\mathscr{D}}{\mathscr{D} t}+6 D\right) \boldsymbol{\sigma}^{\prime}=2 \mu \Phi\left\{\left(\frac{\mathscr{D}}{\mathscr{D} t}+6 D\right)\left(\frac{5}{2}+\frac{26}{147} \epsilon^{2}\right) \mathbf{E}+\frac{18}{5} \epsilon^{2} D \mathbf{E}\right\}
$$

These limiting forms illustrate the general ability of anisotropic fluid models to reproduce the same asymptotic forms as the so-called isotropic (simple fluid) models in the limit of small deformation rates, provided only that the anisotropy is induced by the flow and is not inherent in the material at rest. To the extent that the non-Newtonian features of some materials, such as the suspension under present consideration, are a result of induced microstructural anisotropy, we submit that the anisotropic fluid theories should be preferred to the more common 'isotropic' simple fluid theories. 


\section{Shear flow}

The time-dependent two-dimensional simple shear flow

$$
u=\gamma(t) y, \quad v=w=0
$$

offers a variety of interesting types of material deformation histories, many of which correspond closely to experimental conditions. The cases that we shall consider in this section are the sudden application of a steady shear, which will also yield the constant-shear solution, the sudden ending of a steady shear, an oscillatory shear for weak flow strengths and finally an arbitrary strength steady shear on which is superimposed a small oscillation. Bird et al. (1971) have recently studied a number of these same examples for a suspension of dumbbell-shaped particles in the limit of weak shear flow (strong Brownian motion) $|\gamma| \mid D \ll 1$

To solve (5) for the components of $\mathbf{A}$, the particular form of the flow gradient is substituted. This yields a single and two coupled first-order ordinary differential equations, together with one redundant relationship expressing the traceless property of A. It is convenient to eliminate the trace condition by the introduction of a new variable $\Delta$, such that

$$
A_{11}=-\frac{1}{2} A_{33}+\Delta, \quad A_{22}=-\frac{1}{2} A_{33}-\Delta .
$$

The differential equations can be solved explicitly:

$$
\begin{aligned}
& \begin{array}{r}
A_{12}(t)=A_{12}(0) e^{-6 D t} \cos \left(\int_{0}^{t} \gamma d \tau\right)-\Delta(0) e^{-6 D t} \sin \left(\int_{0}^{t} \gamma d \tau\right) \\
+\frac{3}{2} \int_{0}^{t} e^{-6 D(t-\tau)} \gamma(\tau) \cos \left(\int_{\tau}^{t} \gamma d \tau^{\prime}\right) d \tau
\end{array} \\
& \Delta(t)=\Delta(0) e^{-6 D t} \cos \left(\int_{0}^{t} \gamma d \tau\right)+A_{12}(0) e^{-6 D t} \sin \left(\int_{0}^{t} \gamma d \tau\right) \\
& +\frac{3}{2} \int_{0}^{t} e^{-6 D(t-\tau)} \gamma(\tau) \sin \left(\int_{\tau}^{t} \gamma d \tau^{\prime}\right) d \tau \\
& A_{13}(t)=A_{13}(0) e^{-6 D t} \cos \left(\int_{0}^{t} \frac{1}{2} \gamma d \tau\right)+A_{23}(0) e^{-6 D t} \sin \left(\int_{0}^{t} \frac{1}{2} \gamma d \tau\right), \\
& \begin{array}{l}
A_{23}(t)=A_{23}(0) e^{-6 D t} \cos \left(\int_{0}^{t} \frac{1}{2} \gamma d \tau\right)-A_{13}(0) e^{-6 D t} \sin \left(\int_{0}^{t} \frac{1}{2} \gamma d \tau\right), \\
A_{33}(t)=A_{33}(0) e^{-6 D t} .
\end{array}
\end{aligned}
$$

The connexion between this solution and that obtained from the calculus of the Jaumann derivative for more general flows should be apparent. The appearance of the exponential factor $e^{-6 D t}$ in these expressions means that the orientation state becomes independent of the initial conditions (as specified by $\mathbf{A}(0)$ ) as time advances. In the sense of continuum mechanics, this suspension has a fading memory for past configurations. 
In shear flow, equation (6) for the particle stress reduces to

$$
\left.\begin{array}{rl}
\sigma_{12}^{\prime} & =\Phi \mu \gamma\left\{\frac{5}{2}+\epsilon^{2}\left[\frac{26}{147}+\frac{12}{5} \frac{D}{\gamma} A_{12}-\frac{1}{7} A_{33}\right]\right\}, \\
\sigma_{11}^{\prime}-\sigma_{33}^{\prime} & =\Phi \mu \gamma \epsilon^{2}\left\{\frac{6}{5} \frac{D}{\gamma}\left(2 \Delta-3 A_{33}\right)+\frac{2}{7} A_{12}\right\}, \\
\sigma_{22}-\sigma_{33}^{\prime} & =\Phi \mu \gamma \epsilon^{2}\left\{-\frac{6}{5} \frac{D}{\gamma}\left(2 \Delta+3 A_{33}\right)+\frac{2}{7} A_{12}\right\}, \\
\sigma_{13}^{\prime} & =\Phi \mu \gamma \epsilon^{2}\left\{\frac{12}{5} \frac{D}{\gamma} A_{13}+\frac{1}{7} A_{23}\right\}, \\
\sigma_{23}^{\prime} & =\Phi \mu \gamma \epsilon^{2}\left\{\frac{12}{5} \frac{D}{\gamma} A_{23}+\frac{1}{7} A_{13}\right\} .
\end{array}\right\}
$$

Normal stress differences are employed in order to eliminate the arbitrary isotropic pressure.

\subsection{The starting problem}

As a first example of a time-dependent shear, we consider the response of the suspension to a suddenly imposed shear flow of constant magnitude,

$$
\gamma(t)= \begin{cases}0 & t<0 \\ \gamma \quad t \geqslant 0\end{cases}
$$

where $\gamma$ is a constant. As can be seen in (7), the immediate response of the material will depend on the initial orientation state of the suspension. Here we suppose that the particle orientations are completely random at the initial instant, corresponding to the state that will inevitably be attained in a quiescent suspension owing to the randomizing effect of Brownian rotations. Hence

$$
\mathbf{A}=\mathbf{0} \text { at } t=0 .
$$

With these initial conditions the solution (7) takes the form

$$
\begin{aligned}
A_{13} & =A_{23}=A_{33}=0, \\
A_{12} & =\frac{3}{2} \frac{\beta}{1+\beta^{2}}\left[\left(1-e^{-6 D t} \cos \gamma t\right)+\beta e^{-6 D t} \sin \gamma t\right], \\
\Delta & =\frac{3}{2} \frac{\beta}{1+\beta^{2}}\left[\beta\left(1-e^{-6 D t} \cos \gamma t\right)-e^{-6 D t} \sin \gamma t\right],
\end{aligned}
$$

and the particle contribution to the bulk stress is

$$
\left.\begin{array}{rl}
\sigma_{12}^{\prime} & =\Phi \mu \gamma\left\{\frac{5}{2}+\epsilon^{2}\left[\frac{26}{147}+\frac{3}{5} \frac{1}{1+\beta^{2}}\left(\left(1-e^{-6 D t} \cos \gamma t\right)+\beta e^{-6 D t} \sin \gamma t\right)\right]\right\}, \\
\sigma_{11}^{\prime}-\sigma_{33}^{\prime} & =\Phi \mu \gamma \epsilon^{2} \frac{3}{35} \frac{1}{1+\beta^{2}}\left[12 \beta\left(1-e^{-6 D t} \cos \gamma t\right)+\left(5 \beta^{2}-7\right) e^{-6 D t} \sin \gamma t\right], \\
\sigma_{22}^{\prime}-\sigma_{33}^{\prime} & =\Phi \mu \gamma \epsilon^{2} \frac{3}{35} \frac{1}{1+\beta^{2}}\left[-2 \beta\left(1-e^{-6 D t} \cos \gamma t\right)+\left(5 \beta^{2}+7\right) e^{-6 D t} \sin \gamma t\right], \\
\sigma_{13}^{\prime} & =\sigma_{23}^{\prime}=0,
\end{array}\right\}
$$


where the shear strength is non-dimensionalized with the Brownian diffusion coefficient $\beta=\gamma / 6 D$.

The material response at large times is precisely that described previously for steady shear. The effective viscosity is shear-thinning with constant limiting values for large and small shear rates. This shear thinning can be interpreted in terms of the microstructural dynamies. The degree of alignment of the particles with the direction of the flow increases with the shear rate. In such an orientation, the particles cause less disruption to the basic flow. The corresponding normal stresses are non-zero and unequal. They increase quadratically in $\gamma$ for small shear rates $(\beta \ll 1)$, and finally tend to constant limits (as $\beta \gg 1$ ) which are independent of $\gamma$. This predicted behaviour is qualitatively similar to actual experimental results in a variety of polymeric solutions and melts, though, of course, the magnitudes and other quantitative features are not reproducod because of obvious differences between the microstructure of these materials and that of the dilute suspension considered here.

While the details of the transient response depend upon the magnitude of the shear rate $\gamma$ and the diffusion coefficient $D$, several general features may be noted. First, the material response is characterized by two natural time scales, $(6 D)^{-1}$ and $\gamma^{-1}$, arising respectively from the diffusive and advective aspects of the microscale particle motion. Second, the transients are oscillatory with frequency $\gamma$ and are exponentially damped with increasing time. For example, the effective viscosity, defined as the ratio of the shear stress to the shear rate, starts with the value

$$
\left.\mu^{*}\right|_{0}=\mu\left\{1+\Phi\left[\frac{5}{2}+\frac{26}{147} \epsilon^{2}\right]\right\}
$$

attains a maximum value

$$
\left.\mu^{*}\right|_{\max }=\left.\mu^{*}\right|_{0}+\Phi \mu \epsilon^{2} \frac{3}{5} \frac{1}{1+\beta^{2}}\left(1+\beta e^{-\pi / 2 \beta}\right)
$$

after a time $t=\pi / 2 \gamma$ and thereafter oscillates with decreasing amplitude about the final steady-state value

$$
\left.\mu *\right|_{\infty}=\left.\mu^{*}\right|_{0}+\Phi \mu \epsilon^{2} \frac{3}{5} \frac{1}{1+\beta^{2}} .
$$

The initial value is that expected in the absence of Brownian rotations and can be attributed to the instantaneously established rotation of the suspended particles. The oscillatory behaviour, including the overshoot phenomenon, is generated by the oscillatory bulk strain experienced by the particles as they execute their Jeffrey orbits. The temporal development on the scale $(6 D)^{-1}$ arises from the change of the distribution due to the diffusive action of rotary Brownian movements. It should also be noted that for a given material the overshoot in the shear stress is enhanced by higher shear rates and in the limit tends to a constant value of $\frac{18}{5} \Phi \mu D \epsilon^{2}$. Similar transient behaviour has been observed (Booij 1966; Vinogradov \& Belkin 1965) in a variety of polymeric materials. In particular Vinogradov \& Belkin (1965) found in polymer melts an overshoot in the shear stress whose magnitude increased with $\gamma$, and which occurred at a time that decreased with increasing $\gamma$. 
In contrast to the shearing stress, the transverse and streamwise normal stress differences are initially zero because of the isotropic orientation at the start. Non-zero normal stress differences are established in an oscillatory manner as the orientation distribution becomes anisotropic. In the steady state, the Weissenberg. hypothesis (which assumes that the secondary normal stress difference vanishes) is approximated in a numerical sense, because

$$
\sigma_{11}^{\prime}-\sigma_{33}^{\prime}=6\left(\sigma_{33}^{\prime}-\sigma_{22}^{\prime}\right) \text { as } t \rightarrow \infty \text {. }
$$

However, at intermediate times the stress is dominated by the oscillatory component $\sin \gamma t$, and hence the normal stress differences can be of similar magnitude (and sign).

\subsection{The stopping problem}

The second example, closely related to the first, involves the sudden termination of a steady shear flow,

$$
\gamma(t)= \begin{cases}\gamma & (t<0), \\ 0 & (t \geqslant 0) .\end{cases}
$$

The initial conditions for $\mathbf{A}$ will be taken as the steady-state values obtained above. The solution (7) then becomes

$$
\begin{aligned}
A_{13} & =A_{23}=A_{33}=0, \\
A_{12} & =\frac{3}{2} \frac{\beta}{1+\beta^{2}} e^{-6 D t}, \\
\Delta & =\frac{3}{2} \frac{\beta^{2}}{1+\beta^{2}} e^{-6 D t}
\end{aligned}
$$

where $\beta$ is again the non-dimensional shear rate $\gamma / 6 D$. Substituting these into the expressions (8) for the stress yields

$$
\left.\begin{array}{rl}
\sigma_{12}^{\prime} & =\Phi \mu \gamma \epsilon^{2} \frac{3}{5} \frac{1}{1+\beta^{2}} e^{-6 D t} \\
\sigma_{11}^{\prime}-\sigma_{33}^{\prime} & =\sigma_{33}^{\prime}-\sigma_{22}^{\prime}=\Phi \mu \gamma \epsilon^{2} \frac{3}{5} \frac{\beta}{1+\beta^{2}} e^{-6 D t}
\end{array}\right\}
$$

Thus the preferred alignment and the stress relax exponentially with a characteristic time scale $(6 D)^{-1}$. The stress also suffers, at the initial instant, an immediate reduction to a small fraction of its value in steady shear. This reflects the fact that the contribution due to the particle motion about the Jeffrey orbits vanishes instantaneously (when microscale fluid and particle inertia are neglected). Alternatively we may note that the suspension is nearly Newtonian, and that the Newtonian stress vanishes with the shear.

Experimental observations in polymeric solutions and melts (see e.g. Huppler et al. 1967) again show qualitatively similar behaviour in that the shear stress relaxes more quickly than the normal stresses. It is also observed that the stress relaxes more rapidly when the preceding shear is stronger, and this is not reflected in our expressions. As we shall see, this is one of the few characteristics of experimental materials which is not qualitatively described by the near-sphere. 
suspension. We can only speculate that the more efficient relaxation from higher flow strengths may reflect a difference in the relative excitation of several modes which take a spectrum of decay times.

\subsection{Oscillatory weak shear}

As a third example, we consider an oscillatory simple shear flow

$$
\gamma(t)=\gamma \sin \omega t
$$

Our primary interest here is the material response after a time sufficiently long that the transients can be ignored, leaving only the stationary time-dependent response. For this large-time behaviour the coefficients $A_{i j}$ are independent of the initial orientation state. The magnitude of the flow strength will be restricted by

$$
\gamma \ll \max (\omega, D) .
$$

We introduce the non-dimensional frequency and shear rate

$$
\alpha=\omega / 6 D, \quad \beta=\gamma / 6 D
$$

to obtain the following simplified expressions for the solution (7):

$$
\begin{gathered}
A_{13}=A_{23}=A_{33}=0, \\
A_{12}=\frac{3}{2} \beta(\sin \omega t-\alpha \cos \omega t) /\left(1+\alpha^{2}\right)+O(\beta /(1+\alpha))^{3}, \\
\Delta=\frac{3}{4} \beta^{2}\left[1-\left(3 \alpha \sin 2 \omega t+\left(1-2 \alpha^{2}\right) \cos 2 \omega t\right) /\left(1+4 \alpha^{2}\right)\right] /\left(1+\alpha^{2}\right)+O(\beta /(1+\alpha))^{4} .
\end{gathered}
$$

Substituting these formulae into (8), the particle stress is easily shown to be

$$
\left.\begin{array}{l}
\sigma_{12}^{\prime}=\Phi \mu \gamma\left\{\frac{5}{2} \sin \omega t+\epsilon^{2}\left[\frac{26}{147} \sin \omega t+\frac{3}{5}(\sin \omega t-\alpha \cos \omega t) /\left(1+\alpha^{2}\right)\right]\right\} \\
\sigma_{11}^{\prime}-\sigma_{33}^{\prime}=\Phi \mu \gamma \epsilon^{2} \frac{3}{35} \beta\left\{6-\left[\left(5 \alpha^{2}+78\right) \alpha \sin 2 \omega t+\left(3 \alpha^{2}+6\right) \cos 2 \omega t\right] /\right. \\
\left.\left(1+4 \alpha^{2}\right)\right\} /\left(1+\alpha^{2}\right), \\
\sigma_{22}^{\prime}-\sigma_{33}^{\prime}=\Phi \mu \gamma \epsilon^{2} \frac{3}{35} \beta\left\{-1+\left[\left(10 \alpha^{2}-8\right) \alpha \sin 2 \omega t+\left(17 \alpha^{2}-1\right) \cos 2 \omega t\right] /\right. \\
\left.\left(1+4 \alpha^{2}\right)\right\} /\left(1+\alpha^{2}\right) .
\end{array}\right\}
$$

It is customary to discuss the shear stress in terms of a complex viscosity $\eta^{\prime}-i \eta^{\prime \prime}$ defined by

Hence

$$
\begin{gathered}
\sigma_{12}(t)=\gamma\left[\eta^{\prime} \sin \omega t-\eta^{\prime \prime} \cos \omega t\right] . \\
\eta^{\prime}=\mu\left\{1+\Phi\left[\frac{5}{2}+\frac{28}{14 \frac{7}{4}} \epsilon^{2}+\frac{3}{5} \epsilon^{2} /\left(1+\alpha^{2}\right)\right]\right\} \\
\eta^{\prime \prime}=\mu \Phi \epsilon^{2} \times \frac{3}{5} \alpha /\left(1+\alpha^{2}\right) .
\end{gathered}
$$

The real part of the intrinsic viscosity decreases monotonically as the frequency of the shear flow increases, whereas the imaginary part exhibits a maximum at an intermediate $\omega$, tending to zero in both the limits $\omega \rightarrow 0$ or $\infty$. The existence of a positive $\eta^{\prime \prime}$ results from the memory of the microstructure: this term represents the phase lag of the orientation state in adapting to the changing strain field. The reduction in the magnitude of the viscosity as the frequency increases may be interpreted as a decrease in alignment of the particles in the direction of maximum strain (and therefore disruption to the flow), because in a rapidly oscillating system there is insufficient time for the orientations to change. 
The normal stress differences show both a steady and an oscillatory component, the frequency of the latter being twice that of the basic imposed flow. Note that it is possible for the normal stress differences to change sign for part of the cycle.

A relatively large number of experimental investigations of this oscillatory shear have been carried out, many of which are summarized by Ferry (1970). We call particular attention to the recent works of Ferry, Holmes, Lamb \& Matheson (1966), Moore, McSkimin, Gieniewski \& Andreatch (1969), Endo \& Nagasawa (1970) and finally, Wales \& den Otter (1970). They found that the shear stresses oscillate with the same frequency as the applied shear flow, and have a magnitude proportional to the applied shear amplitude. Furthermore, the dynamic viscosity $\eta^{\prime}$ is found to decrease from a constant value at low frequencies to a non-zero but smaller value in the limit as $\omega \rightarrow \infty$. In addition, a maximum in the component $\eta^{\prime \prime}$ is also observed at an intermediate value of $\omega$. Finally, the normal stress differences are observed to consist of a steady part, plus an oscillatory component with twice the applied frequency whose magnitude is proportional to the square of the applied shear. This behaviour is qualitatively identical to the predicted behaviour in the near-sphere suspension.

One of the reasons for the concentrated experimental effort with oscillatory shear has been the suggestion, by various investigators, of a relation between the dynamic stress variation with frequency $\omega$ and the steady stress variation with shear rate $\gamma$. The existence of such a relationship would clearly be of considerable significance since it would allow one to use dynamic measurements as a function of frequency to get steady shear rheological information, especially in the range of large $\gamma$ where the steady shear experiments are difficult. Unfortunately, it is only in the opposite limit that the relationships are well-established (see Wales \& den Otter). A comparison of the stress distributions (9) and (11) shows the following exact relations for a suspension of near-spheres at arbitrary $\gamma$ and $\omega$ :

$$
\begin{gathered}
\left.\mu^{*}(\beta)\right|_{s}=\left.\eta^{\prime}(\alpha)\right|_{o, \alpha=\beta}, \\
\left.\left(\sigma_{11}^{\prime}-\sigma_{33}^{\prime}\right)(\beta)\right|_{s}=\left.6\left(\sigma_{33}^{\prime}-\sigma_{22}^{\prime}\right)(\beta)\right|_{s}=\left.\frac{12}{7} \eta^{\prime \prime}(\alpha) \gamma\right|_{o, \alpha=\beta},
\end{gathered}
$$

where the subscripts $s$ and $o$ imply 'steady' and 'oscillating' respectively. Coleman \& Markovitz (1964) suggested a normal stress relationship which is equivalent to part of our expression. They expected it to be valid only for low shear rates which in our problem means for $\beta(=\alpha) \ll 1$. Our expressions, on the other hand, are valid for all $\beta(=\alpha)$.

\subsection{Slightly unsteady shear}

The final example of a time-dependent unidirectional shear that we consider is a steady flow on which is superimposed small oscillations in time. The applied shear is given by

$$
\gamma(t)=\gamma(1+\kappa \sin \omega t)
$$

As in the previous case, we find it necessary to restrict attention here to small magnitudes of the oscillation, $\kappa \ll 1$, so that we can use a perturbation expression in the small parameter $\kappa$ to achieve approximate solutions for the coefficients $A_{i j}$. We are primarily interested in the long-time stationary behaviour of the system, when these coefficients will be independent of the initial orientation state. 
Using the long-time solutions to (7) correct to $O\left(\kappa^{2}\right)$, the asymptotic expansion of the particle stress is found to be

$$
\begin{aligned}
& \sigma_{12}^{\prime}=\Phi \mu \gamma\left\{\left\{\frac{5}{2}+\epsilon^{2}\left[\frac{26}{147}+\frac{3}{5} \frac{1}{1+\beta^{2}}\right]\right\}\right. \\
& +\kappa\left\{\left[\frac{5}{2}+\epsilon^{2}\left[\frac{26}{147}+\frac{3}{10} \frac{1}{\alpha}\left(\frac{\beta+\alpha}{1+(\beta+\alpha)^{2}}-\frac{\beta-\alpha}{1+(\beta-\alpha)^{2}}\right)\right]\right] \sin \omega t\right. \\
& \left.+\epsilon^{2} \frac{3}{10} \frac{1}{\alpha}\left[\frac{2 \beta^{2}}{1+\beta^{2}}-\frac{(\beta-\alpha)^{2}}{1+(\beta-\alpha)^{2}}-\frac{(\beta+\alpha)^{2}}{1+(\beta+\alpha)^{2}}\right] \cos \omega t\right) \\
& +\kappa^{2} \epsilon^{2} \frac{3}{40} \frac{\beta}{\alpha}\left\{2\left[\frac{\beta-\alpha}{1+(\beta-\alpha)^{2}}+\frac{\beta+\alpha}{1+(\beta+\alpha)^{2}}-\frac{2 \beta}{1+\beta^{2}}\right]\right. \\
& \left.+2 \text { nd harmonics }\}+O\left(\kappa^{3}\right)\right\} \text {, } \\
& \sigma_{11}^{\prime}-\sigma_{33}^{\prime}=\Phi \mu \gamma \epsilon^{2}\left\{\left[\frac{36}{35} \frac{\beta}{1+\beta^{2}}\right]+\kappa \frac{3}{70} \frac{1}{\alpha}\left\{\left[\frac{10 \beta \alpha}{1+\beta^{2}}-\frac{(12 \beta-7 \alpha)(\beta-\alpha)}{1+(\beta-\alpha)^{2}}\right.\right.\right. \\
& \left.+\frac{(12 \beta+7 \alpha)(\beta+\alpha)}{1+(\beta+\alpha)^{2}}\right] \sin \omega t \\
& +\left[\frac{2 \beta \alpha}{1+\beta^{2}}\left(5 \beta^{2}-7\right)+\frac{\left(7+5 \alpha \beta-5 \beta^{2}\right)(\beta-\alpha)}{1+(\beta-\alpha)^{2}}\right. \\
& \left.\left.\left.+\frac{\left(7+5 \alpha \beta+5 \beta^{2}\right)(\beta+\alpha)}{1+(\beta+\alpha)^{2}}\right] \cos \omega t\right\}+O\left(\kappa^{2}\right)\right\} \\
& \sigma_{22}^{\prime}-\sigma_{33}^{\prime}=\Phi \mu \gamma \epsilon^{2}\left\{\left[-\frac{6}{35} \frac{\beta}{1+\beta^{2}}\right]+\kappa \frac{3}{70} \frac{1}{\alpha}\left\{\left[\frac{10 \beta \alpha}{1+\beta^{2}}-\frac{(7 \alpha-2 \beta)(\beta-\alpha)}{1+(\beta-\alpha)^{2}}\right.\right.\right. \\
& \left.-\frac{(2 \beta+7 \alpha)(\beta+\alpha)}{1+(\beta+\alpha)^{2}}\right] \sin \omega t \\
& +\left[\frac{2 \beta \alpha}{1+\beta^{2}}\left(5 \beta^{2}+7\right)-\frac{\left(7-5 \alpha \beta+5 \beta^{2}\right)(\beta-\alpha)}{1+(\beta-\alpha)^{2}}\right. \\
& \left.\left.\left.+\frac{\left(7-5 \alpha \beta-5 \beta^{2}\right)(\beta+\alpha)}{1+(\beta+\alpha)^{2}}\right] \cos \omega t\right\}+O\left(\kappa^{2}\right)\right\} \text {. }
\end{aligned}
$$

The particle contribution to the bulk shear stress oscillates about a constant value

$$
\Phi \mu \gamma\left\{\frac{5}{2}+\epsilon^{2}\left[\frac{26}{147}+\frac{3}{5} \frac{1}{1+\beta^{2}}+\kappa^{2} \frac{3}{20} \frac{\beta}{\alpha}\left(\frac{\beta-\alpha}{1+(\beta-\alpha)^{2}}+\frac{\beta+\alpha}{1+(\beta+\alpha)^{2}}-\frac{2 \beta^{2}}{1+\beta^{2}}\right)\right]\right\},
$$

which differs only at $O\left(\kappa^{2}\right)$ from the steady flow value $(\kappa=0)$. Hence, it may be seen that the presence of the oscillating shear does produce a slight $O\left(\kappa^{2}\right)$ shear thinning in the steady component of shear stress, which results from the average strain seen by the non-uniformly rotating particles. The effects of an increasing rate of steady shear upon the steady component of stress, and of an increasing frequency of oscillating shear upon the oscillating component of stress, have already been noted as shear-thinning in previous sections and these features are preserved in the present example. The effect of increasing steady shear rate upon the oscillating shear stresses at $O(\kappa)$ is also to decrease their effective viscosity 
(cf. previous case). This is caused by the particle alignment in the direction of the flow created by the steady shear. Examination of the sign of the out-of-phase shear stress component reveals the very interesting prediction that the oscillating shear stress may either lead or lag the applied shear flow depending on whether $\alpha^{2}$ is smaller or larger than $3 \beta^{2}-1$. When the flow is sufficiently strong, $\beta>3^{-\frac{1}{2}}$, $\alpha^{2}$ can be smaller than $3 \beta^{2}-1$ at lower frequencies. This phenomenon can be explained by the fading memory of the microstructure rotating with the vorticity of the basic steady flow.

The normal stresses oscillate slightly, at $O(\kappa)$, about the steady shear values with the frequency of the imposed oscillations. This should be contrasted with the second-harmonic oscillations of the normal stress in the absence of the steady component of the shear (cf. previous case). Second-harmonic terms would arise at $O\left(\kappa^{2}\right)$ in the present calculation but we have not exhibited them in order to maintain a reasonable degree of brevity.

As with the other problems presented so far, a number of experimental investigations of polymer rheology have used an oscillating shear superimposed on a steady shear. Particularly noteworthy are the investigations of Booij (1966, 1968). Booij found that in the linear range the steady part of the shear stress was unchanged by the small oscillations, and that the oscillating part had a magnitude proportional to $\kappa$ and a frequency equal to that of the applied oscillations. The steady shear caused a decrease in the effective viscosity seen by the oscillating shear, and the phase of the shear stress showed the phenomenon of leading the shear rate at the lower frequencies. The normal stresses exhibited a steady part owing to the steady shear, and in the linear range, a component which oscillated with the imposed frequency. Finally, all the stress components also showed second-harmonic oscillations as $\kappa$ became sufficiently large. All of these measured characteristics are qualitatively similar to the behaviour of our simple nearsphere suspension.

\section{Axisymmetric pure straining motions}

The next class of examples contains unidirectional elongational flows defined by

$$
u=-\frac{1}{2} E(t) x, \quad v=-\frac{1}{2} E(t) y, \quad w=E(t) z .
$$

There is no vorticity in this flow so the near-sphere analysis will be restricted by $\left|E\left(r^{2}-1\right) /\left(r^{2}+1\right) D\right| \ll 1$. We shall investigate two start-up problems in this section: the Heaviside start-up to a constant strain rate, and the ramp start-up corresponding to a strain rate which increases linearly with time. The large-time limit of the Heaviside start-up yields the constant strain rate solution.

With no vorticity in the flow, the governing problem (5) reduces to five independent first-order ordinary differential equations together with one redundant relation. For the sake of economy, we shall confine attention to axially symmetric orientation distributions, and therefore axially symmetric stresses. The solution (5) is thence

$$
A_{i j}=0, \quad i \neq j ; \quad A_{11}=A_{22}=-\frac{1}{2} A, \quad A_{33}=A,
$$


where

$$
A(t)=A(0) e^{-6 D t}+\int_{0}^{t} e^{-6 D(t-\tau)} 3 E(\tau) d \tau .
$$

The particle contribution to the stress $(6)$ is

where

$$
\begin{gathered}
\sigma_{i j}^{\prime}=0, \quad i \neq j ; \quad \sigma_{11}^{\prime}=\sigma_{22}^{\prime}=-\frac{1}{2} \sigma^{\prime}, \quad \sigma_{33}^{\prime}=\sigma^{\prime}, \\
\sigma^{\prime}=2 \Phi \mu\left\{\frac{5}{2} E+\epsilon^{2}\left[\frac{26}{147} E+\frac{6 D}{5} A+\frac{1}{7} A E\right]\right\} .
\end{gathered}
$$

It should be noted that for all initial conditions the stress tensor must eventually take the Newtonian form in this class of flows (although the effective viscosity will vary).

\subsection{The starting problem}

The starting-up problem for the elongational flow

$$
E(t)= \begin{cases}0 & (t<0) \\ E & (t \geqslant 0)\end{cases}
$$

with random initial conditions $(A=0, t<0)$ yields for $t \geqslant 0$

and

$$
\begin{gathered}
A(t)=\frac{1}{2} \frac{E}{D}\left(1-e^{-6 D t}\right) \\
\sigma^{\prime}(t)=2 \Phi \mu E\left\{\frac{5}{2}+\epsilon^{2}\left[\left(\frac{571}{735}+\frac{E}{14 D}\right)-\left(\frac{3}{5}+\frac{E}{14 D}\right) e^{-6 D t}\right]\right\} .
\end{gathered}
$$

The effective viscosity $\mu^{*}$ increases monotonically in time from its initial value of

$$
\left.\mu^{*}\right|_{0}=\mu\left[1+\Phi\left(\frac{5}{2}+\frac{26}{147} \epsilon^{2}\right)\right]
$$

to the final steady-state value of

$$
\left.\mu^{*}\right|_{\infty}=\mu\left[1+\Phi\left(\frac{5}{2}+\frac{571}{735} \epsilon^{2}+\frac{E}{14 D} \epsilon^{2}\right)\right]
$$

in a characteristic time of $(6 D)^{-1}$. The steady effective viscosity shows the familiar strain thickening, with a linear increase for increases in $E / D$.

The non-Newtonian effects have been confined to be of $O\left(\epsilon^{2}\right)$ in our formulation (5) and (6), i.e. the above results are limited to $|E \epsilon| D \mid \ll 1$. It is not difficult to find that when $|E \epsilon| D \mid \gg 1$ the steady effective viscosity tends to a constant value

$$
\left.\mu^{*}\right|_{\infty}= \begin{cases}\mu\left[1+\Phi\left(\frac{5}{2}+\frac{5}{7} \epsilon\right)\right] & \text { if } E \epsilon>0 \\ \mu\left[1+\Phi\left(\frac{5}{2}-\frac{5}{14} \epsilon\right)\right] & \text { if } E \epsilon<0 .\end{cases}
$$

It can be further shown that the steady effective viscosity takes, at arbitrary values of $\epsilon E / D$ (but $\epsilon \rightarrow 0$ ), the form

where

$$
\left.\mu^{*}\right|_{\infty}=\mu\left\{1+\Phi\left[\frac{5}{2}-\frac{80}{441} \epsilon^{2}+\left[\frac{10}{7} \epsilon-\frac{893}{441} \epsilon^{2}+\frac{12 \epsilon^{2}}{x}\right] \frac{J^{\prime}}{J}+\frac{3160}{441} \epsilon^{2} \frac{J^{\prime \prime}}{J}\right]\right\}
$$

The strain thickening is a result of the preferential alignment of the particles along the principal axis of the strain. In that direction the particles reach out, on 
average, into the largest relative flow, and so through the no-slip condition create the greatest net disturbance. This strain-thickening mechanism should generalize to other materials with a similar microstructure that can be preferentially aligned. Indeed recent experimental measurements of polymeric materials (Metzner \& Metzner 1970; Astarita \& Nicodena 1970) do show such a qualitative behaviour.

\subsection{The starting problem with linearly increasing strain}

A second, related flow is the start with a ramp function strain. One reason for the interest in this flow is that pure straining motions naturally produced are not usually steady: one would like to determine the extent to which the elongational viscosity for steady flow is applicable to time-dependent cases. We suppose that the suspension is initially in a state of random orientation. Then with

$$
E(t)=f t, \quad(t \geqslant 0)
$$

we find

$$
A(t)=(f t / 2 D)\left[1-\left(1-e^{-6 D t}\right) / 6 D t\right],
$$

hence yielding the bulk stress

$$
\sigma^{\prime}=2 \Phi \mu E(t)\left\{\frac{5}{2}+\epsilon^{2}\left[\left(\frac{571}{735}+\frac{E(t)}{14 D}\right)-\left(\frac{3}{5}+\frac{E(t)}{14 D}\right)\left(1-e^{-6 D t}\right) / 6 D t\right]\right\} .
$$

Comparing (16) and (15) we see that, for the particular time variation we have chosen, the time-dependent effective viscosity is slightly less than that given for a steady strain equal to the instantaneous magnitude. This is simply a result of the memory of the microstructure interacting with the nonliner strain-thickening mechanism outlined earlier: there is a lag as the orientation state adapts to the changing flow. At $D t$ becomes large (but $|\epsilon f t| D \mid \ll 1$ ), the transients decay away leaving an increasing effective viscosity which is less than the steady strain value by $\mu \epsilon^{2} f / 84 D^{2}$ (and this is small compared with the instantaneously evaluated strain thickening $\left.\mu \epsilon^{2} f t / 14 D\right)$. We conclude therefore that the strain thickening will be common amongst time-dependent flows which do not change rapidly on the time scale $(6 D)^{-1}$.

\section{Slightly wobbly shear}

A rheologically interesting flow which is closely related to the slightly unsteady shear described earlier is the flow composed of super-imposed orthogonal shear flows studied experimentally by Simmons (1970):

$$
u=\gamma y, \quad v=0, \quad w=\gamma y \kappa \sin \omega t, \quad \gamma=\text { constant } .
$$

We shall be interested in the long-time behaviour of the oscillating system. The governing problem (5) does not reduce and remains five coupled first-order linear ordinary differential equations with varying coefficients. In order to simplify the problem we must restrict attention to small amplitude oscillations, $\kappa \ll 1$. We expand the coefficients $A_{i j}$ in an asymptotic series in the small parameter $\kappa$. With the frequency and shear strength non-dimensionalized by the relaxation rate $6 D$,

$$
\alpha=\omega / 6 D, \quad \beta=\gamma / 6 D,
$$


the particle stress to $O\left(\kappa^{2}\right)$ is

$$
\begin{aligned}
& \sigma_{12}^{\prime}=\Phi \mu \gamma\left\{\frac{5}{2}+\epsilon^{2}\left[\left(\frac{26}{147}+\frac{3}{5} \frac{1}{1+\beta^{2}}\right)+\kappa^{2}\left(\Sigma \frac{-7 \beta^{3}-28 \beta+10 \beta^{4} s+20 \beta^{2} s-4 s}{1+\left(\frac{1}{2} \beta+s\right)^{2}}\right)\right.\right. \\
& \left.\left.+2 \text { nd harmonics })+O\left(\kappa^{4}\right)\right]\right\} \\
& \sigma_{13}^{\prime}=\Phi \mu \gamma \epsilon^{2} \kappa \frac{3}{280} \frac{1}{1+\beta^{2}}\left\{\left[20 \beta+\Sigma \frac{\frac{19}{2} \beta^{3}+38 \beta+9 \beta^{2} s+28 s}{1+\left(\frac{1}{2} \beta+s\right)^{2}}\right] \sin \omega t\right. \\
& \left.+\left[\Sigma \frac{S}{|S|}-\frac{\frac{5}{2} \beta^{4}-3 \beta^{2}+28-5 \beta^{3} s-24 \beta s}{1+\left(\frac{1}{2} \beta+s\right)^{2}}\right] \cos \omega t+O\left(\kappa^{2}\right)\right\} \\
& \sigma_{23}^{\prime}=\Phi \mu \gamma \kappa\left\{\left[\frac{5}{2}+\epsilon^{2}\left[\frac{26}{147}+\frac{3}{280} \frac{1}{1+\beta^{2}}\right.\right.\right. \\
& \left.\left.\times\left(-20 \beta^{2}+\Sigma \frac{\frac{5}{2} \beta^{4}+17 \beta^{2}+28+5 \beta^{3} s-4 \beta s}{1+\left(\frac{1}{2} \beta+s\right)^{2}}\right)\right]\right] \sin \omega t \\
& \left.-\frac{3}{280} \frac{1}{1+\beta^{2}}\left(\Sigma \frac{S}{|S|} \frac{\frac{9}{2} \beta^{3}+18 \beta+19 \beta^{2} s+28 s}{1+\left(\frac{1}{2} \beta+s\right)^{2}}\right) \cos \omega t+O\left(\kappa^{2}\right)\right\}, \\
& \sigma_{11}^{\prime}-\sigma_{33}^{\prime}=\Phi \mu \gamma \epsilon^{2} \frac{36}{35} \frac{\beta}{1+\beta^{2}}\left\{1-\kappa^{2}\left[\frac{1}{16} \frac{1}{1+\beta^{2}} \Sigma \frac{\frac{5}{2} \beta^{4}+9 \beta^{2}+4-\beta^{3} s}{1+\left(\frac{1}{2} \beta+s\right)^{2}}\right.\right. \\
& \left.+2 \text { nd harmonics }]+O\left(\kappa^{4}\right)\right\} \text {, } \\
& \sigma_{22}^{\prime}-\sigma_{33}^{\prime}=\Phi \mu \gamma \epsilon^{2} \frac{6}{35} \frac{\beta}{1+\beta^{2}}\left\{1+\kappa^{2}\left[\frac{1}{16} \frac{1}{1+\beta^{2}} \Sigma \frac{6 \beta^{4}+21 \beta^{2}+28-14 \beta^{2} s-32 s}{1+\left(\frac{1}{2} \beta+s\right)^{2}}\right.\right. \\
& \left.+2 \text { nd harmonies }]+O\left(\kappa^{4}\right)\right\}
\end{aligned}
$$

where the summation is taken over $s=-\alpha$ and $s=+\alpha$. The second harmonics have not been given as they are difficult to interpret.

The shear stress $\sigma_{12}^{\prime}$ and the two normal stress differences are changed from their values in a steady simple shear (cf. (9)) only at $O\left(\kappa^{2}\right)$. The shear stress and primary normal stress difference are decreased while the secondary difference is increased by the presence of the oscillating transverse shear. Comparing the shear stress in a weakly oscillating simple shear, equation (11), with the transverse oscillating shear stress $\sigma_{23}^{\prime}$, one finds that the basic steady shear reduces the complex effective viscosity seen by the transverse motions. The interaction of the two shear fields produces a novel stress component $\sigma_{13}^{\prime}$. A full physical interpretation of these phenomena in terms of the microstructural dynamics is complicated in this genuinely three-dimensional time-dependent motion. We look forward to experimental results in this bidirectional shear flow, as no quantitative information is yet available to compare with our near-sphere suspension results.

\section{Maxwell orthogonal rheometer}

A non-viscometric flow which has been used in experimental rheology is

$$
u=\Omega z+\gamma y, \quad v=0, \quad w=-\Omega x .
$$


This is a simple shear $\gamma y$ combined with a vorticity $2 \Omega$ about the gradient direction. Here we shall only consider the steady response to a steady flow. The governing system (5) then simplifies to a fifth-order linear algebraic set of equations. With non-dimensional parameters

$$
\alpha=\Omega / 6 D, \quad \beta=\gamma / 6 D, \quad \delta=\left(4+20 \alpha^{2}+5 \beta^{2}+8 \alpha^{2} \beta^{2}+16 \alpha^{4}+\beta^{4}\right)^{-1},
$$

the solution becomes

$$
\begin{aligned}
& A_{12}=\frac{3}{2} \beta \delta\left(4+16 \alpha^{2}+\beta^{2}\right), \quad A_{23}=-3 \alpha \beta \delta\left(2+8 \alpha^{2}-\beta^{2}\right), \\
& A_{13}=-9 \alpha \beta^{2} \delta, \quad A_{11}=\frac{3}{2} \beta^{2} \delta\left(4+4 \alpha^{2}+\beta^{2}\right), \\
& A_{22}=-\frac{3}{2} \beta^{2} \delta\left(4+16 \alpha^{2}+\beta^{2}\right), \quad A_{33}=18 \alpha^{2} \beta^{2} \delta .
\end{aligned}
$$

The particle stress $(6)$ now yields

$$
\left.\begin{array}{rl}
\sigma_{12}^{\prime} & =\Phi \mu \gamma\left\{\frac{5}{2}+\epsilon^{2}\left[\frac{26}{147}+\frac{3}{35} \delta\left(28+112 \alpha^{2}+7 \beta^{2}-30 \alpha^{2} \beta^{2}\right)\right]\right\}, \\
\sigma_{23}^{\prime} & =-\Phi \mu \gamma \epsilon^{2} \frac{3}{35} \alpha \delta\left(28+112 \alpha^{2}+\beta^{2}\right), \\
\sigma_{13}^{\prime} & =-\Phi \mu \gamma \epsilon^{2} \frac{3}{35} \alpha \beta \delta\left(52+40 \alpha^{2}-5 \beta^{2}\right), \\
\sigma_{11}^{\prime}-\sigma_{33}^{\prime} & =\Phi \mu \gamma \epsilon^{2} \frac{36}{35} \beta \delta\left(4+2 \alpha^{2}+\beta^{2}\right), \\
\sigma_{22}^{\prime}-\sigma_{33}^{\prime} & =-\Phi \mu \gamma \epsilon^{2} \frac{6}{35} \beta \delta\left(4+58 \alpha^{2}+\beta^{2}\right) .
\end{array}\right\}
$$

In the limit $\alpha \rightarrow 0$ we recover the steady shear flow form (9) for the stress pattern. Comparing (18) with (9), we see the additional rotation $(\Omega \neq 0)$ causes a number of changes. First, the shear stress $\sigma_{12}^{\prime}$ is reduced and, for certain combinations of $\alpha$ and $\beta$, the effective viscosity can take values less than the steady $(\alpha=0)$ high shear rate $(\beta \rightarrow \infty)$ limit. Second, the primary normal stress difference $\sigma_{11}^{\prime}-\sigma_{33}^{\prime}$ is decreased while the secondary difference $\sigma_{22}^{\prime}-\sigma_{33}^{\prime}$ becomes more negative. Eventually when $\alpha$ is large enough the magnitude of the secondary difference exceeds that of the primary difference. Finally, novel stresses $\sigma_{13}^{\prime}$ and $\sigma_{23}^{\prime}$ are produced. All these effects should be interpreted as a net rotation through less than a quadrant by the additional vorticity $2 \Omega$ of any preferential alignment.

In the limit $\beta \rightarrow 0$, we recover some aspects of the weakly oscillating shear flow (11). As is noted in another theoretical model by Bird \& Harris (1968, 1970), the shear stresses $\sigma_{12}^{\prime}$ and $\sigma_{23}^{\prime}$ are then given by the real and imaginary parts of the complex effective viscosity evaluated for the frequency $\omega=\Omega$, i.e.

$$
\sigma_{12}^{\prime} \sim \gamma \eta^{\prime}(\alpha), \quad \sigma_{23}^{\prime} \sim-\gamma \eta^{\prime \prime}(\alpha) \quad \text { for } \beta \rightarrow 0
$$

The connexion with the oscillating shear (for weak flow strengths) is explained in our orientation model by the oscillating flow field a particle must experience as it rotates with the vorticity $2 \Omega$. Thus the phase lag in time has become a phase lag in direction. The effect of a non-zero shear strength is to thin both components of the complex viscosity, as would be expected from increased preferential alignment in the flow direction.

An experimental study of a polymer melt by Maxwell \& Chartoff (1965) in such a rheometer did reveal a number of the qualitative features contained in (18). The shear viscosity $\sigma_{12} / \gamma$ did show a thinning separately due to increases in the vorticity and the shear strength, $\alpha$ and $\beta$. The orthogonal shear stress $\sigma_{13}$ was 
found to be linear in the shear strength (presumably only at low $\beta$ ) with a fre-quency dependence which vanished in both limits $\alpha \rightarrow 0, \infty$, with an intermediate maximum. Our model only fails to yield a normal stress that is linear in the shear, but one should be sceptical of such an experimental observation, which apparently depends on the sign of the shear.

\section{Conclusions}

In the preceding sections of this paper we derived a general expression governing the rheological behaviour of a dilute suspension of nearly spherical particles subject to Brownian couples and have considered the application of this constitutive relation to the description of a suspension's response for several specific imposed flow fields. As a result of this and previous investigations we may characterize the rheological behaviour as follows.

(i) An exponentially fading memory with a characteristic time scale of $(6 D)^{-1}$. When there is a vorticity $2 \Omega$ present, the response may oscillate with frequencies $2 \Omega$ and $\Omega$ owing to the rotation of the microstructure.

(ii) A nonlinear effect of a strain-thickening viscosity in a uniaxial extensional motion, although the final constant limiting value of the viscosity at high strain rates is not contained within our model equations (5) and (6).

(iii) A nonlinear effect of a shear-thinning viscosity in a shear flow, with constant limiting values of the viscosity at small and large shear rates. There is also a thinning action of increasing frequency in oscillatory shear and of increasing steady shear strength and/or frequency on a superimposed oscillatory shear (parallel or transverse).

(iv) A nonlinear effect of unequal non-zero normal stress differences in shear flow. These are quadratic in the shear rate for weak flows but become constant for strong flows.

\section{REFERENCES}

Astarita, G. \& Nicodema, L. 1970 Chem. Engng J. 1, 57.

BAtChelor, G. K. 1970 J. Fluid Mech. 41, 545.

Btrd, R. B. \& HARRIS, E. K. 1968 A.I.Ch.E. J. 14, 758.

BIrD, R. B. \& HARrTs, E. K. 1970 A.I.Ch.E. J. 16, 149.

Bird, R. B., Warner, H. R. \& Evans, D. C. 1971 Adv. Polym. Sci. 8, 1.

BoEder, P. 1932 Z. Phys. 75, 258.

Boot, H. C. 1966 Rheologica Acta, 5, 215, 222.

Boot, H. C. 1968 Rheologica Acta, 7, 202.

Bretherton, F. P. 1962 J. Fluid Mech. 14, 284.

Burgers, J. M. 1938 In Second Report on Viscosity and Plasticity, Chapter 3. Kon. Ned. Akad. Wet. Verhand. (Eerste Sectie), 16, 113.

Coleman, B. D. \& Markovitz, H. 1964 J. Appl. Phys. 35, 1.

Endo, H. \& Nagasawa, M. 1970 J. Polym. Sei. 8, 371.

Ferry, J. D. 1970 Viscoelastic Properties of Polymers. Wiley.

Ferry, J. D., Holmes, L. A., Lamb, J. \& Matheson, A. J. 1966 J. Phys. Chem. 70, 1685.

Frankel, N. A. \& Acrivos, A. 1970 J. Fluid Mech. 44, 65.

GIESEKUS, H. 1962 Rheologica Acta, 2, 50.

Goddard, J. D. \& Miller, C. 1966 Rheologica Acta, 5, 177. 
GOddard, J. D. \& Miller, C. 1967 J. Fluid Mech. 28, 657.

H.INCH, E. J. \& LEAL, L. G. 1972 J. Fluid Mech. 52, 683.

Huppler, J. D., MacDonald, T. F., Ashare, E., Spriggs, T. W., Bird, R. B. \& Holmes, L. A. 1967 Trans. Soc. Rheol. 11, 181.

JefFery, G. B. 1922 Proc. Roy. Soc. A 102, 161.

Kirkwood, J. G. \& AUer, P. L. 1951 J. Chem. Phys. 19, 281.

Kirkwood, J. G. \& Plock, R. J. 1956 J. Chem. Phys. 24, 665.

LEAL, L. G. \& Hinch, E. J. 1971 J. Fluid Mech. 46, 685.

LeAL, L. G. \& Hinch, E. J. 1972 Rheologica Acta, to appear.

Maxwell, B. \& Chartoff, R. P. 1965 Trans. Soc. Rheol. 9 (1), 41.

Metzner, A. B. \& Metzner, A. P. 1970 Rheologica Acta, 9, 174.

Moore, R. S., MaSkimin, H. J., Gieniewski, C. \& Andreatch, P. 1969 J. Chem. Phys. 50, 5088 .

Peterlun, A. 1938 Z. Phys. 111, 232.

Peterlin, A. \& Struart, H. A. 1939 Z. Phys. 112, 1, 129.

Sadron, Ch. 1953 Flow Properties of Disperse Systems (ed. J. J. Hermans), chap. 4. North-Holland Pub. Co.

Scheraga, H. A. 1955 J. Chem. Phys. 23, 1526.

Simmons, J. M. 1970 Rheologica Acta, 7, 184.

Stewart, W. E. \& Sørensen, J. P. 1972 Trans. Soc. Rheol. 16 (1), 1.

Takserman-Krozer, R. \& Ziabicki, A. $1963 \mathrm{~J}$. Polym. Sci. A 1, 491, 507.

Vinogradov, G. V. \& Belkin, I. M. 1965 J. Polym. Sci. 3, 917.

WALES, J. L. S. \& DEN OtTer, J. L. 1970 Rheologica Acta, 9, 115. 